\title{
Beyond packing of hard spheres: The effects of core softness, non-additivity, intermediate-range repulsion, and many-body interactions on the glass-forming ability of bulk metallic glasses
}

\author{
Kai Zhang*, ${ }^{1,2}$ Meng Fan*, ${ }^{1,2}$ Yanhui Liu, ${ }^{1,2}$ Jan Schroers, ${ }^{1,2}$ Mark D. Shattuck, ${ }^{3,1}$ and Corey S. O'Hern ${ }^{1,2,4,5}$ \\ ${ }^{1}$ Department of Mechanical Engineering and Materials Science, \\ Yale University, New Haven, Connecticut, 06520, USA \\ ${ }^{2}$ Center for Research on Interface Structures and Phenomena, \\ Yale University, New Haven, Connecticut, 06520, USA \\ ${ }^{3}$ Department of Physics and Benjamin Levich Institute, \\ The City College of the City University of New York, New York, New York, 10031, USA \\ ${ }^{4}$ Department of Physics, Yale University, New Haven, Connecticut, 06520, USA \\ ${ }^{5}$ Department of Applied Physics, Yale University, New Haven, Connecticut, 06520, USA
}

(Dated: January 11, 2018)

\begin{abstract}
When a liquid is cooled well below its melting temperature at a rate that exceeds the critical cooling rate $R_{c}$, the crystalline state is bypassed and a metastable, amorphous glassy state forms instead. $R_{c}$ (or the corresponding critical casting thickness $d_{c}$ ) characterizes the glass-forming ability (GFA) of each material. While silica is an excellent glass-former with small $R_{c}<10^{-2} \mathrm{~K} / \mathrm{s}$, pure metals and most alloys are typically poor glass-formers with large $R_{c}>10^{10} \mathrm{~K} / \mathrm{s}$. Only in the past thirty years have bulk metallic glasses (BMGs) been identified with $R_{c}$ approaching that for silica. Recent simulations have shown that simple, hard-sphere models are able to identify the atomic size ratio and number fraction regime where BMGs exist with critical cooling rates more than 13 orders of magnitude smaller than those for pure metals. However, there are a number of other features of interatomic potentials beyond hard-core interactions. How do these other features affect the glass-forming ability of BMGs? In this manuscript, we perform molecular dynamics simulations to determine how variations in the softness and non-additivity of the repulsive core and form of the interatomic pair potential at intermediate distances affect the GFA of binary alloys. These variations in the interatomic pair potential allow us to introduce geometric frustration and change the crystal phases that compete with glass formation. We also investigate the effect of tuning the strength of the many-body interactions from zero to the full embedded atom model on the GFA for pure metals. We then employ the full embedded atom model for binary BMGs and show that hard-core interactions play the dominant role in setting the GFA of alloys, while other features of the interatomic potential only change the GFA by one to two orders of magnitude. Despite their perturbative effect, understanding the detailed form of the intermetallic potential is important for designing BMGs with $\mathrm{cm}$ or greater casting thickness.
\end{abstract}

PACS numbers: 64.70.pe,64.70.Q-,61.43.Fs,61.66.Dk,61.43.Dq

\section{INTRODUCTION}

When metallic liquids are cooled at rates $R$ exceeding the critical cooling rate $R_{c}$, crystallization can be bypassed and amorphous alloys are formed [1]. Pure metals and most alloys are extremely poor glass formers with $R_{c}>10^{10} \mathrm{~K} / \mathrm{s}$. In contrast, a number of bulk metallic glasses (BMGs) have been identified with $R_{c}<1 \mathrm{~K} / \mathrm{s}$ and critical casting thickness $d_{c}>1 \mathrm{~cm}$, which enables them to be employed in commercial applications [2, 3]. The discovery of novel BMGs with optimized casting thickness and mechanical properties has largely been a trialand-error process [4, 5]. Although combinatorial deposition and characterization techniques [6, 7] now allow efficient exploration of parameter space, there are an exponentially large number of possible BMG-forming atomic compositions [8]. Thus, a quantitative and predictive

\footnotetext{
* These authors contributed equally to this work.
}

understanding of the GFA of BMG-forming alloys is necessary to narrow down the vast parameter space.

Silica and polymers possess critical cooling rates that are more than 15 and 10 orders of magnitude lower, respectively, than those for pure metals (Fig. 1). Network bonding in silica and chain entanglement in polymers provide the physical mechanisms to inhibit crystallization [16 18]. In contrast, the main source of geometric frustration in alloys is the mismatch between atomic sizes [19 24]. Molecular dynamics simulations of binary hard spheres have shown that tuning the atomic size ratio can decrease $R_{c}$ by more than 13 orders of magnitude [15]. Packing of hard spheres can also rationalize the correlation between the number of components, their atomic size ratios, and the GFA of BMGs [8].

Although the packing of hard spheres plays an important role in determining the GFA of alloys, it is obvious that metals possess additional features that are not represented by hard-sphere interactions. Other features of metallic interactions, such as metallic bonding [25], the form of the interatomic pair potential, and many- 


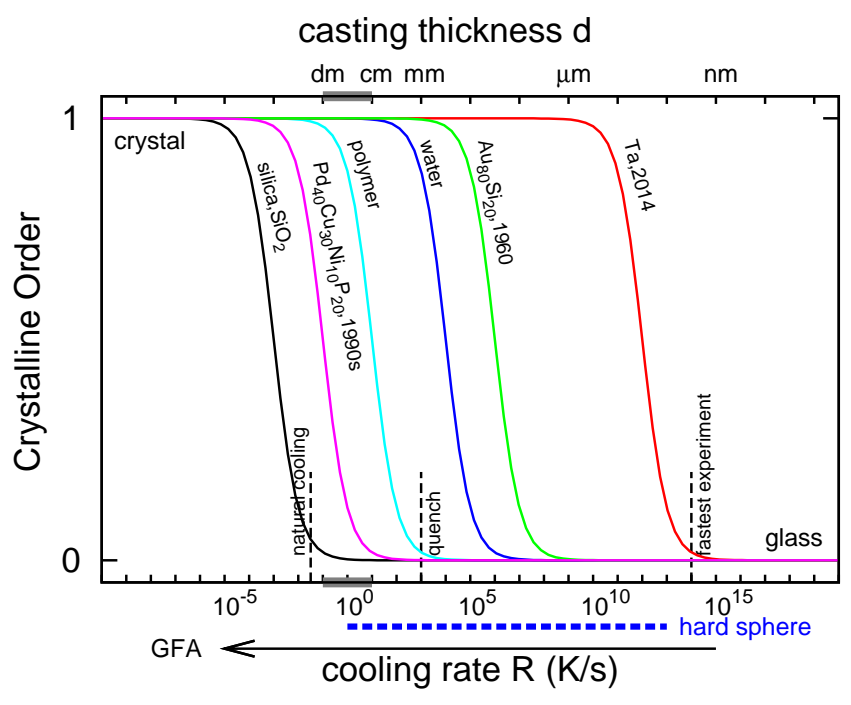

FIG. 1: Schematic diagram of crystalline order (such as bond orientational order [9]) versus the cooling rate $R$ in $\mathrm{K} / \mathrm{s}$ for several materials. The critical cooling rate $R_{c}$ at which there is a rapid rise in the crystalline order is inversely correlated with the material's critical casting thickness $d_{c}$. Smaller $R_{c}$ (and larger $d_{c}$ ) indicate enhanced glass-forming ability (GFA). Pure metals, e.g. Ta, are extremely poor glass formers [3]. The GFA of the first fabricated metallic alloy $\mathrm{Au}_{80} \mathrm{Si}_{20}$ [10] is similar to that of water [11], but is a poor glass-former compared to polymers [12] and silica [13]. The best bulk metallic glasses (BMGs), e.g. $\mathrm{Pd}_{40} \mathrm{Cu}_{30} \mathrm{Ni}_{10} \mathrm{P}_{20}$ [14], possess $\mathrm{cm}$ or greater critical casting thicknesses and $<1 \mathrm{~K} / \mathrm{s}$ critical cooling rates (solid gray bars). In recent simulations, we have shown that hard-core atomic interactions can account for more than 13 orders of magnitude variation in $R_{c}$ (thick dashed line) from $10^{0} \mathrm{~K} / \mathrm{s}$ for typical BMGs to $10^{13} \mathrm{~K} / \mathrm{s}$ for pure metals [15].

body interactions [26], can change the crystalline structure that competes with glass formation and change the prediction of $R_{c}$ by several orders of magnitude from the hard-sphere value. Compared to the $\sim 13$ orders of magnitude variation in $R_{c}$ that results from the packing of hard-spheres, changes to $R_{c}$ are small, but not negligible and may explain the crucial differences between an amorphous film and a bulk metallic glass. Since the critical casting thickness $d_{c}$ is negatively correlated with $R_{c}$ and increasing $R_{c}$ by two orders of magnitude can reduce $d_{c}$ by one order of magnitude [27], more accurate models of intermetallic potentials are needed to identify BMGs with $d_{c}>1 \mathrm{~cm}$ (Fig. 1).

The interatomic potential in the embedded atom model (EAM) is frequently implemented in computational studies of the structural and mechanical properties, as well as the dynamics, of metallic systems [26]. The EAM potential energy includes a pairwise-additive term, which is in general different from the hard-sphere and Lennard-Jones pair potentials (Fig. 2 (a)), and a many-body contribution from the electron charge den- sity, which is fitted to $a b$ initio calculations of lattice parameters, elastic constants, and other thermodynamic properties [28, 29].

In this manuscript, we seek to identify the key features of the pairwise and many-body interactions that strongly influence the GFA of alloys. For example, we investigate the effects of the softness of the pairwise repulsive core, pairwise non-additivity, and the form of the pairwise intermediate-range repulsion on the GFA. We then measure the GFA for the full embedded atom models of several pure metals and BMGs to determine the contribution of the many-body interactions on the GFA. We find that the changes in the GFA arising from variations in the pair and many-body contributions of the embedded atom model are small compared to the 13 orders of magnitude change in GFA between monoatomic and binary and ternary hard-sphere systems. However, these peturbations to the GFA may still be important for discovering new bulk metallic glass formers.

The manuscript includes three additional sections after the introduction. First, in Sec. 2. we describe the hard-sphere, repulsive Lennard-Jones, Lennard-Jones, and Dzugutov-Shi potentials used to vary the form and non-additivity of the pairwise interactions. We also introduce the embedded atom model for pure metals and alloys. For each interatomic potential, we discuss the methods employed to measure the critical cooling rate $R_{c}$. We then report the results for the GFA for all interaction potentials in Sec. 3. We conclude the manuscript in Sec. 4

\section{MODELS AND METHODS}

As described above, the embedded atom model for metallic systems includes pairwise and many-body interactions. In this section, we define three metrics (core softness, non-additivity, and intermediate-range repulsion) to characterize the form of the pairwise interactions. We describe molecular dynamics simulations of monodipserse and binary systems interacting via generalized Lennard-Jones or Dzugutov-Shi [30, 31] potentials to quantify the effects of the softness of the repulsive core and strength of the intermediate-range repulsion on the GFA. We also introduce molecular dynamics simulations of binary hard spheres to study variations in the GFA from non-additive pairwise interactions. We estimate values for the pairwise core softness, non-additivity, and form of the intermediate-range repulsive interactions from fits to the pairwise contributions of the EAM for pure metals and binary BMGs. We also introduce the Lennard-Jones and full EAM potentials that we employ to study the effects of many-body interactions on the GFA. 

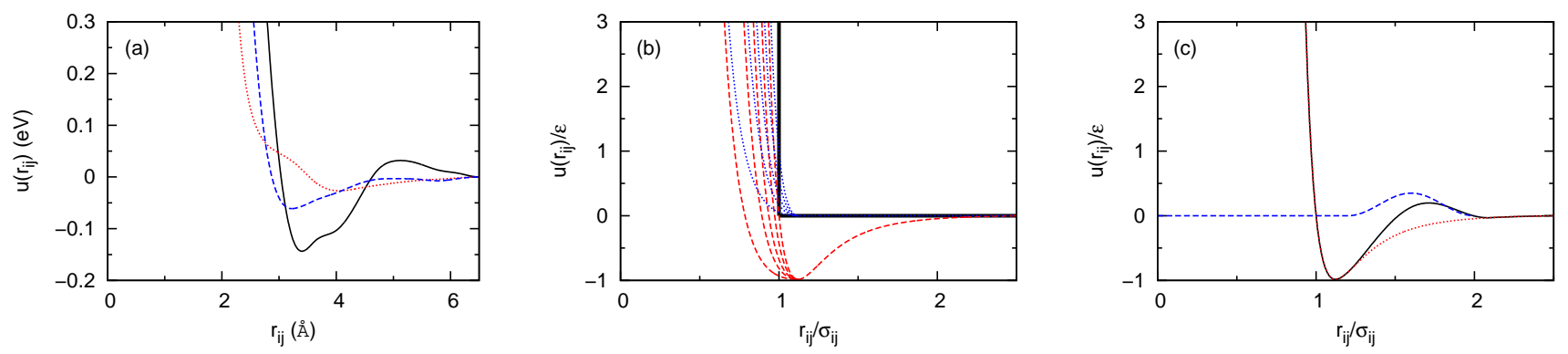

FIG. 2: (a) The pairwise potentials $u\left(r_{i j}\right)$ (in eV) as a function of interatomic separation $r_{i j}$ for $\mathrm{Zr}-\mathrm{Zr}$ (solid line), Cu-Cu (dotted line), and $\mathrm{Zr}-\mathrm{Cu}$ (dashed line) interactions for the embedded atom model for Zr-Cu alloys [29]. (b) Generalized Lennard-Jones (Eq. 1) (dashed lines) and repulsive Lennard-Jones (Eq. 2) (dotted lines) interatomic potentials for several values of the core softness exponent $m=1,3,5,8$, and 12 (from left to right) compared to the hard-sphere potential (thick solid line). (c) Dzugutov-Shi interatomic potential (Eq. 6) (solid line) decomposed into the Lennard-Jones (dotted line) and sinusoidal "bump" potentials (dashed line).

\subsection{Lennard-Jones (LJ) and Repulsive Lennard-Jones (RLJ) Potentials}

tential (Fig. 2 (b)),

To tune the softness of the pairwise repulsive core [32], we employ the generalized $m-n$ Lennard-Jones (LJ) po-

$$
u_{\mathrm{LJ}}^{m-n}\left(r_{i j}\right)=\left\{\begin{array}{cl}
\epsilon\left[2^{\frac{m}{6}} \frac{n}{m-n}\left(\frac{\sigma_{i j}}{r_{i j}}\right)^{m}-2^{\frac{n}{6}} \frac{m}{m-n}\left(\frac{\sigma_{i j}}{r_{i j}}\right)^{n}\right], & r_{i j} \leq r_{m} \\
u_{\mathrm{LJ}} & , r_{i j}>r_{m}
\end{array}\right.
$$

where $\sigma_{i j}=\left(\sigma_{i}+\sigma_{j}\right) / 2, \sigma_{i}$ is the diameter of atom $i$, and $\epsilon$ is the energy scale of the interaction. The interaction potential has a minimum $u_{m}=-\epsilon$ at $r_{m}=2^{1 / 6} \sigma_{i j}$. The exponent $m$ (or equivalently the curvature $\kappa$ of the pair potential at the minimum) controls the softness of the repulsive core, where smaller $m$ corresponds to softer interactions. Note that the generalized Lennard-Jones potential is fixed at $u_{\mathrm{LJ}}\left(r_{i j}\right) \equiv u_{\mathrm{LJ}}^{12-6}\left(r_{i j}\right)$ for $r_{i j}>r_{m}$. To separate the effects of the attractive interactions from the repulsive core, we also studied the generalized $m-n$ repulsive Lennard-Jones (RLJ) potential [33] as shown in Fig. 2(b):

$$
u_{\mathrm{RLJ}}^{m-n}\left(r_{i j}\right)=\left\{\begin{array}{cl}
u_{\mathrm{LJ}}^{m-n}\left(r_{i j}\right)+\epsilon, & r_{i j} \leq r_{m} \\
0 & , r_{i j}>r_{m}
\end{array}\right.
$$

To obtain physical values for the softness exponent $m$, we fit the repulsive part of the EAM pair potential of typical BMG-forming elements to $u_{\mathrm{RLJ}}^{m-6}(r)$. As shown in Table I. we find that $m$ varies from approximately 3 to 14. The repulsive cores for most metals appear softer than Lennard-Jones interactions with $m=12$.

To investigate the effects of softness of the pairwise repulsive core on the GFA of metallic systems, we performed molecular dynamics (MD) simulations of $N=$ 1372 spherical atoms with mass $m_{0}$ that interact via the generalized Lennard-Jones and repulsive Lennard-Jones potentials with $n=6$ and a range of $m$ values. We studied three binary LJ systems with softness exponents $m_{A}=m_{B}=m_{A B}=12(\mathrm{LJ} 12-6), m_{A}=m_{B}=m_{A B}=5$ (LJ5-6), and $m_{A}=12, m_{B}=5$, and $m_{A B}=8$ (LJ12-6/LJ5-6). We set the atomic diameter ratio to be $\alpha=\sigma_{B} / \sigma_{A}=0.95$ and varied the number fraction of small atoms $x_{B}=N_{B} / N$ from 0 to 1 . Temperatures and times are given in units of $\epsilon / k_{B}$ and $\sigma_{A} \sqrt{m_{0} / \epsilon}$, respectively. After equilibrating the systems at high temperature $T_{0}=2$, the liquids were cooled exponentially $T(t)=T_{0} \exp (-R t)$ with rate $R$ to low temperature, $T_{f}=0.01$, using the Gaussian constraint thermostat [43] with time step $\Delta t=0.001$. Constant volume $V$ simulations at number density $\rho \sigma_{A}^{3}=N \sigma_{A}^{3} / V=1$ were performed for both the LJ and RLJ models. For LJ systems, we also cooled systems with the constraint that the pressure $p$ (in units of $\epsilon / \sigma_{A}^{3}$ ) decreased exponentially in time from an initial pressure $p_{0}=1$ to final pressure $p_{f}=0.001$ according to

$$
p(t)=p_{0} \exp \left[-\frac{\log \left(p_{0} / p_{f}\right)}{\log \left(T_{0} / T_{f}\right)} R t\right]
$$

using a Gaussian constraint barostat 43]. A cooling rate of $R=1$ in the units used in the MD simulations corre- 
TABLE I: Softness exponent $m$ from the repulsive Lennard-Jones potential (Eq. 2) for the self-part of the pair potential contribution to the embedded atom model for common atomic species found in BMGs 29]. The exponent $m$ varies linearly with the curvature $\kappa$ (given in units of $\epsilon / \sigma_{A}^{2}$ ) of the interatomic potential at its minimum $r_{m}$. References for the EAM potentials are provided in columns 4 and 8 . Several atom types possess multiple EAM potentials.

\begin{tabular}{|c|c|c|c|c|c|c|c|}
\hline atom & $\kappa$ & $m$ & Ref. & atom & $\kappa$ & $m$ & Ref. \\
\hline \multirow[t]{3}{*}{$\mathrm{Zr}$} & 38.77 & 8.14 & [29] & $\mathrm{Pb}$ & 35.30 & 7.41 & {$[34]$} \\
\hline & 31.77 & 6.67 & [34] & $\mathrm{Mg}$ & 42.69 & 8.97 & [34] \\
\hline & 66.91 & 14.05 & [28] & $\mathrm{Fe}$ & 31.41 & 6.60 & [34] \\
\hline \multirow[t]{3}{*}{$\mathrm{Ag}$} & 48.77 & 10.24 & [29] & $\mathrm{Co}$ & 32.88 & 6.90 & [34] \\
\hline & 35.49 & 7.45 & [34] & & 38.06 & 7.99 & 35 \\
\hline & 40.35 & 8.47 & [36] & $\mathrm{Ta}$ & 21.36 & 4.49 & 34 \\
\hline \multirow[t]{3}{*}{$\mathrm{Al}$} & 33.48 & 7.03 & [29] & & 15.64 & 3.28 & [37] \\
\hline & 20.65 & 4.34 & [34] & & 24.56 & 5.16 & 38 \\
\hline & 10.89 & 2.29 & [39] & $\mathrm{Cu}$ & 28.66 & 6.02 & [34] \\
\hline \multirow[t]{3}{*}{$\mathrm{Ni}$} & 34.36 & 7.21 & [29] & $\mathrm{Au}$ & 38.40 & 8.06 & [34] \\
\hline & 30.66 & 6.44 & [34] & & 48.20 & 10.12 & 40] \\
\hline & 47.96 & 10.07 & [41] & $\mathrm{Ti}$ & 32.93 & 6.92 & [34] \\
\hline \multirow[t]{2}{*}{$\mathrm{Pd}$} & 43.72 & 9.18 & [29] & Mo & 20.42 & 4.29 & [34] \\
\hline & 33.39 & 7.01 & [34] & $\mathrm{W}$ & 22.63 & 4.75 & [34] \\
\hline \multirow[t]{2}{*}{$\mathrm{Pt}$} & 43.47 & 9.13 & [29] & $\mathrm{Nb}$ & 28.19 & 5.92 & [42] \\
\hline & 24.04 & 5.05 & [34] & & & & \\
\hline
\end{tabular}

sponds to a cooling rate of $10^{15} \mathrm{~K} / \mathrm{s}$ using $\sigma_{A} \sim 3 \times 10^{-10}$ $\mathrm{m}, \epsilon / k_{B} \sim 10^{3} \mathrm{~K}$, and molar mass $M \sim 10^{-1} \mathrm{~kg} / \mathrm{mol}$, which are typical values for BMGs 32].

\subsection{Non-additive binary hard spheres}

The sizes of metallic atoms are often estimated from the first peak of the radial distribution function $g(r)$ of crystalline and disordered solids [44]. In binary alloys with species $A$ and $B$, the repulsive core $\sigma_{A B}$ between atoms $A$ and $B$ can differ from the average diameter $\bar{\sigma}_{A B}=\left(\sigma_{A}+\sigma_{B}\right) / 2$. We quantify the non-additivity of the pairwise repulsive core using the parameter

$$
\Sigma=\frac{\sigma_{A B}}{\bar{\sigma}_{A B}}-1 \text {. }
$$

Many binary alloys possess $\Sigma<0$, which indicates that the repulsive core $\sigma_{A B}$ between $A$ and $B$ atoms is smaller than the average diameter. We list $\sigma_{A}, \sigma_{B}, \sigma_{A B}$, and $\Sigma$ for several binary alloys obtained from EAM calculations of $g(r)$ in Table I. Non-additive binary hard spheres have been shown to form exotic crystalline structures, in particular intermetallic compounds [45, 46]. In addition, non-additivity due to bond shortening with $\Sigma<0$ can lead to unusual intermediate-range order in BMGs 4749]. The well-studied Kob-Andersen model for $\mathrm{Ni}_{80} \mathrm{P}_{20}$ glasses also has $\Sigma=-0.149$ [50].

To study the effects of nonadditivity on the GFA, we compressed $N=500$ binary hard spheres with mass $m_{0}$
TABLE II: Atomic diameters $\left(\sigma_{A}\right.$ and $\sigma_{B}$ in $\AA$ ) determined by the first peak of the radial distribution function $g(r)$ obtained from EAM simulations of several binary alloys [29]. We also list $\sigma_{A B}$ from $g(r)$, the diameter ratio $\alpha$, and the non-additivity parameter $\Sigma$.

\begin{tabular}{cccccc}
\hline Alloy & $\sigma_{A}$ & $\sigma_{B}$ & $\sigma_{A B}$ & $\alpha$ & $\Sigma$ \\
\hline $\mathrm{Zr}-\mathrm{Cu}$ & 3.15 & 2.49 & 2.75 & 0.79 & -0.025 \\
$\mathrm{Ni}-\mathrm{P}$ & 2.57 & 2.19 & 2.23 & 0.85 & -0.063 \\
$\mathrm{Zr}-\mathrm{Ni}$ & 3.23 & 2.43 & 2.69 & 0.75 & -0.049 \\
$\mathrm{Zr}-\mathrm{Al}$ & 3.21 & 2.69 & 2.93 & 0.84 & -0.007 \\
$\mathrm{Ag}-\mathrm{Al}$ & 2.87 & 2.69 & 2.69 & 0.94 & -0.032 \\
$\mathrm{Mg}-\mathrm{Cu}$ & 3.11 & 2.47 & 2.69 & 0.79 & -0.05 \\
$\mathrm{Mg}-\mathrm{Ti}$ & 2.97 & 2.77 & 2.99 & 0.93 & 0.042 \\
$\mathrm{Y}-\mathrm{Mg}$ & 3.51 & 3.03 & 3.27 & 0.86 & 0 \\
$\mathrm{Pd}-\mathrm{Si}$ & 2.97 & 2.39 & 2.51 & 0.80 & -0.063 \\
$\mathrm{Zr}-\mathrm{Pt}$ & 3.39 & 2.91 & 2.73 & 0.86 & -0.1333 \\
$\mathrm{Cu}-\mathrm{Ni}$ & 2.51 & 2.45 & 2.49 & 0.98 & 0.004 \\
$\mathrm{Mg}-\mathrm{Al}$ & 2.99 & 2.81 & 2.99 & 0.94 & 0.031 \\
\hline
\end{tabular}

that interact pairwise via

$$
u_{\mathrm{HS}}\left(r_{i j}\right)=\left\{\begin{array}{cl}
\infty, & r_{i j} \leq \sigma_{i j} \\
0, & r_{i j}>\sigma_{i j}
\end{array}\right.
$$

over a range of diameter ratios $\alpha$ and number fractions of the small sphere $x_{B}$ using event-driven MD simulations. We first equilibrated liquid states at packing fraction $\phi=0.25$. To compress the system, we ran the MD simulations at constant volume for a time interval $\tau$, and then compressed the system instantaneously until the closest pair of spheres came into contact [15, 23]. We performed successive compressions until the pressure increased to $10^{3}$, which corresponds to $\left(\phi_{J}-\phi\right) / \phi_{J}<10^{-3}$, where $\phi_{J}$ is the packing fraction at the onset of jamming. We varied the compression rate $R \equiv 1 / \tau$ over 5 orders of magnitude [15]. We report $R$ in units of $\sqrt{k_{B} T / m_{0} \sigma_{A}^{2}}$. Note that in these units $R=1$ corresponds to a cooling rate of $10^{12} \mathrm{~K} / \mathrm{s}$ for alloys $[51]$.

\subsection{Dzugutov-Shi (DZ) potential}

The pair potential of many metallic systems includes intermediate-range repulsive interactions [52] in addition to short-range attractive interactions, which can give rise to intermediate-range positional order [53, 54]. Intermediate-range pairwise repulsive interactions are often modeled using the Dzugutov potential [30, 55 57]. Shi et. al. introduced a modified version of the original Dzugutov potential that allows one to continuously tune the interaction potential between the LJ potential to one that includes intermediate-range repulsion [31]. The Dzugutov-Shi (DZ) potential is given by

$$
u_{\mathrm{DZ}}\left(r_{i j}\right)=u_{\mathrm{LJ}}\left(r_{i j}\right)+u_{\mathrm{bump}}\left(r_{i j}\right) \text {, }
$$


TABLE III: Values of the parameters $\xi, \lambda$, and $\delta$ (Eq. 6) that describe the strength and range of the Dzugutov-Shi interatomic potential fit to the self-part of the pair potential of the embedded atom model for several atomic species. The fifth column provides references for the EAM for each atom type.

\begin{tabular}{ccccc}
\hline atom & $\xi(\mathrm{eV})$ & $\lambda$ & $\delta$ & Ref. \\
\hline $\mathrm{Zr}$ & 0.42 & 1.16 & 2.24 & {$[29]$} \\
$\mathrm{Ag}$ & 0.16 & 1.29 & 2.20 & {$[29]$} \\
$\mathrm{Cu}$ & 0.43 & 1.18 & 1.73 & {$[29]$} \\
$\mathrm{Ni}$ & 0.38 & 1.19 & 1.72 & {$[29]$} \\
$\mathrm{Al}$ & 0.10 & 1.76 & 2.35 & {$[29]$} \\
& 0.26 & 1.29 & 1.99 & {$[39]$} \\
$\mathrm{Co}$ & 0.12 & 1.56 & 2.66 & {$[35]$} \\
\hline
\end{tabular}

where the "bump" potential $u_{\text {bump }}\left(r_{i j}\right)$ models the intermediate-range repulsive interactions using a sinusoidal pulse,

$$
u_{\text {bump }}\left(r_{i j}\right)=\left\{\begin{array}{cc}
\xi \sin ^{2}\left(\pi \frac{r_{i j} / \sigma_{i j}-\lambda}{\delta-\lambda}\right) & , \lambda \leq r_{i j} / \sigma_{i j} \leq \delta \\
0 & , \quad \text { otherwise }
\end{array}\right.
$$

of the strength $\xi$ within the range $\lambda \sigma_{i j} \leq r_{i j} \leq \delta \sigma_{i j}$. The location of the peak and width of $u_{\text {bump }}$ are given by $(\lambda+\delta) / 2$ and $\delta-\lambda$. To obtain physical values for $\xi, \lambda$, and $\delta$, we fit the DZ potential to the EAM pair potential for several elements. We show values of $\xi, \lambda$, and $\delta$ for elements commonly found in BMGs in Table III Pb, Pd, Pt, Mg, Fe, Ta, Au, Ti, Mo, W, and $\mathrm{Nb}$ do not have significant intermediate-range repulsive interactions.

To study the effects of intermediate-range repulsive interactions on the GFA, we performed MD simulations of $N=1372$ spherical atoms that interact pairwise via the DZ potential. We followed the same cooling protocol as used for the simulations of Lennard-Jones systems with pressure that decreases exponentially in time as discussed in Sec. 22.1. We fixed the strength of the intermediaterange repulsive interactions at $\xi=0.35 \epsilon$ and varied $\lambda$ and $\delta$ to tune the location of the peak $(\lambda+\delta) / 2$ and range $\delta-\lambda$ of $u_{\text {bump. }}$. We also studied binary mixtures composed of $A$ atoms that interact via the DZ potential with $\xi=0.35 \epsilon, \lambda=1.2$, and $\delta=2.15$, and $B$ atoms that interact via the LJ potential with diameter ratio $\alpha=0.95$. The number fraction of small atoms $x_{B}$ is varied from 0 to 1 in steps of 0.2 .

\subsection{LJ-EAM and EAM potential}

The total potential energy $U$ employed in the embedded-atom model for metals includes pairwise and
TABLE IV: The initial and final temperatures, $T_{i}$ and $T_{f}$, employed during the cooling protocol in the molecular dynamics simulations of the LJ-EAM potential with many-body interaction strength $A$ and electron density inverse decay length $\beta$.

\begin{tabular}{cccccccc}
\hline$A(\mathrm{eV})$ & $\beta\left(\AA^{-1}\right)$ & $T_{i}(K)$ & $T_{f}(K)$ & $A(\mathrm{eV})$ & $\beta\left(\AA^{-1}\right)$ & $T_{i}(K)$ & $T_{f}(K)$ \\
\hline 0 & 4 & 2000 & 300 & 0.66 & 2 & 2000 & 300 \\
1.32 & 2 & 2305 & 343 & 1.98 & 2 & 3285 & 479 \\
0.66 & 4 & 2000 & 300 & 1.32 & 4 & 2257 & 336 \\
1.98 & 4 & 3253 & 475 & 0.66 & 6 & 2000 & 300 \\
1.32 & 6 & 2242 & 337 & 1.98 & 6 & 3271 & 472 \\
\hline
\end{tabular}

many-body contributions:

$$
U=\sum_{i<j} u\left(r_{i j}\right)+\sum_{i} F_{i}\left(\bar{\rho}_{i}^{e}\right)
$$

where the many-body embedding function $F_{i}$ depends on the electron density associated with each atom $i$ (normalized by $\left.e / \sigma_{A}^{3}\right)$ and $\bar{\rho}_{i}^{e}=\sum_{j \neq i} \rho^{e}\left(r_{i j}\right)$ [26, 28, 29]. To quantify the effects of the many-body interactions on the GFA, we focused on the LJ-EAM potential, where $u\left(r_{i j}\right)=u_{L J}\left(r_{i j}\right), F_{i}\left(\bar{\rho}_{i}^{e}\right)=A \bar{\rho}_{i}^{e}\left(\ln \bar{\rho}_{i}^{e}-r_{m} / \sigma_{A}\right) / 2$ and $\rho^{e}\left(r_{i j}\right)=C \exp \left[-\beta\left(r_{i j}-r_{m}\right)\right]$, where $C$ and $r_{m}$ are calibrated to experimental data on alloys [58, 59]. We set the atomic diameter $\sigma_{A}=2.8 \AA$ A and attraction depth $\epsilon=0.2 \mathrm{eV}$ for the LJ potential to match the pair potential of typical metals such as $\mathrm{Zr}$. The parameters $A$ and $\beta$ control the many-body interaction strength and inverse decay length of the electron density, respectively.

We performed MD simulations of the LJ-EAM for several pure metals and of the full EAM for several binary alloys using the LAMMPS simulation software [60]. We cooled systems in the liquid state to low temperature at constant zero pressure at different rates $R$. The initial and final temperatures for several systems (specified by $A$ and $\beta$ ) are summarized in Table IV] For our studies of the full EAM potential, we set $N=4000$ and fixed the initial and final temperatures at $T_{i}=2000 K$ and $T_{f}=300 K$.

\subsection{Critical cooling rate}

To calculate the critical cooling rate $R_{c}$ for each metallic system, we initialized the liquid state at high temperature, cooled the system exponentially to low temperature at a given rate $R$ at either fixed volume or exponentially decaying pressure as in Eq. 3, and measured the global bond orientational order parameter $Q_{6}$ [9]. For hardsphere interactions, we compressed the systems so that the packing fraction approached that at jamming onset exponentially, which is thermodynamically equivalent to cooling systems exponentially [61]. For all systems studied, the average global bond orientational order parameter $Q_{6}$ versus $\log R$ possesses a sigmoidal shape with a midpoint defined by $R_{c}$. Below, we show results for $R_{c}$ 


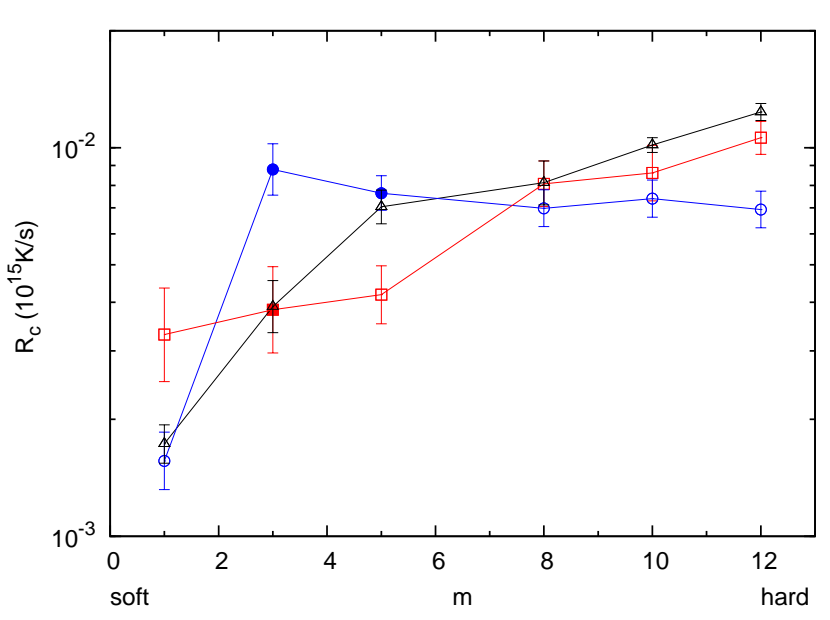

FIG. 3: The critical cooling rate $R_{c}$ (in units of $10^{15} \mathrm{~K} / \mathrm{s}$ ) as a function of the repulsive core softness exponent $m$ in monodisperse systems with generalized repulsive Lennard-Jones (triangles) and Lennard-Jones (squares) interactions cooled at constant density $\rho \sigma_{A}^{3}=1$ and Lennard-Jones interactions with an exponentially decaying pressure given in Eq. 3 (circles). Variations in the softness exponent lead to different crystalline structures that compete with glass formation including face-centered cubic (FCC; empty symbols) and bodycentered cubic (BCC; filled symbols).

for the pair potentials described in Secs. 22.1 22.3 and the full and LJ-EAM potential in Sec. 22.4

\section{RESULTS}

\subsection{Core Softness}

To investigate the effects of softness of the repulsive core on the GFA, we first measured the critical cooling rate $R_{c}$ for monodisperse systems that interact via the generalized LJ (Eq. 1) and RLJ (Eq. 2) pairwise potentials as a function of the softness exponent for $m=1$, 3, 5, 8, 10, and 12. As shown in Fig. 3. when cooling at constant number density $\rho \sigma_{A}^{3}=1$, the GFA increases weakly $\left(R_{c}\right.$ decreases by less than an order of magnitude) as the repulsive core becomes softer ( $m$ decreases). When cooling a LJ system with a pressure that decays exponentially in time as in Eq. 3. the dependence of $R_{c}$ on the softness exponent $m$ is even weaker, except for systems with extremely soft core repulsions with $m=1$. In contrast, most atomic species that are found in BMGs possess $m>4$ (Table $\llbracket$ ).

As shown in Fig. 3, the crystalline structures that compete with glass formation in systems with core-softened RLJ interactions at $\rho \sigma_{A}^{3}=1$ are face-centered cubic (FCC) for all exponents $m$ studied. In addition, FCC crystals compete with glass formation in LJ systems, but as the repulsive core softens, body-centered cubic (BCC) crystals become more stable [62. We find that BCC is

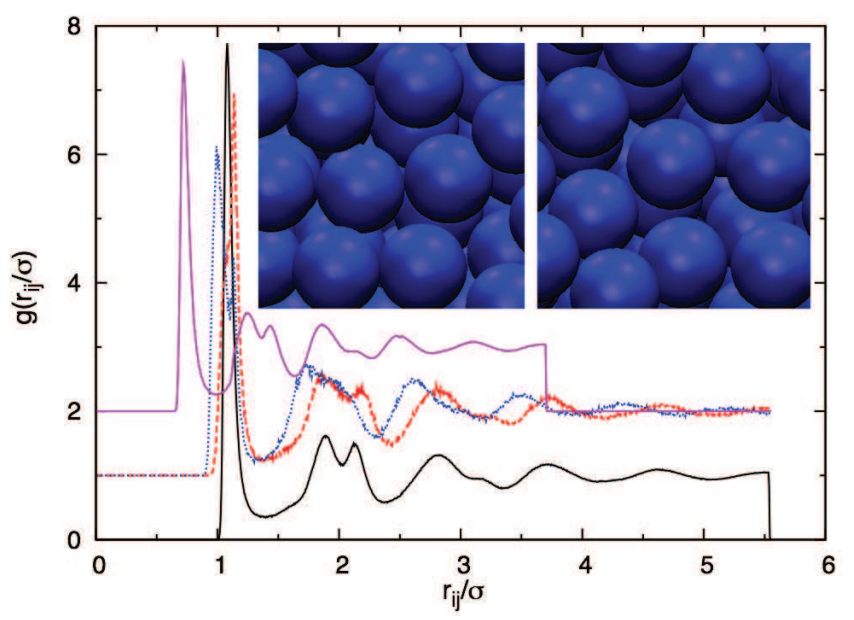

FIG. 4: Radial distribution functions $g\left(r_{i j}\right)$ (vertically shifted for visualization) for monodisperse spherical atoms with diameter $\sigma$ that interact via the generalized $m-6$ LJ potential (Eq. 11) with $m=1$ (top), 3 (middle), and 12 (bottom) cooled at rate $R=0.1>R_{c}$. Compared to the $m=12 \mathrm{LJ}$ system, the soft $m=1 \mathrm{LJ}$ system shows strong volume contraction with the first peak shifted to smaller separations $r_{i j}$. Systems with intermediate softness $m=3$ display two isostructural states: contracted high density (dotted line) and expanded low density (dashed line) glasses. The left and right insets show snapshots of the contracted and expanded $m=3 \mathrm{LJ}$ systems, respectively.

the crystal type that competes with glass formation for $m=3$ LJ systems cooled at constant density $\rho \sigma_{A}^{3}=1$ and for $m=3$ and 5 LJ systems cooled such that the pressure obeys Eq. 3 .

Structural characterizations of atomic systems that interact via the generalized LJ potential are shown in Fig. 4 for cooling rates $R>R_{c}$. As the repulsive core of the potential becomes softer (i.e. $m$ decreases), the attractive well of the potential widens to include secondneighbor attractive interactions, which can compensate repulsive first-neighbor interactions. Indeed, LJ systems with $m=1$ and 3 exhibit phase separation into dilute and compressed regions when cooled at fixed density $\rho \sigma_{A}^{3}=1$ and volume contraction, where the first neighbor separations are smaller than the location of the potential minimum, when cooled such that the pressure obeys Eq. 3 . In fact, the $m=3$ LJ system displays two isostructural glassy states, contracted and expanded, with different densities as shown in the inset to Fig. 4. Similar isostructural transitions have been found in equilibrium systems with narrow-ranged attractive interactions [63]. Large density differences between polymorphs in metallic glasses such as those found in $\mathrm{Ce}_{55} \mathrm{Al}_{45}$ are often attributed to electronic many-body interactions [64]. However, here we show that softening the pairwise repulsive core (which increases the range of the attractive well) can also give rise to polymorphs with different densities.

We also investigated the effects of core softness on the glass-forming ability in binary mixtures that interact via 
the generalized $m$ - 6 LJ potential. We focused on three mixtures with diameter ratio $\alpha=\sigma_{B} / \sigma_{A}=0.95$ : (1) conventional LJ systems with $m=12$, (2) core softened LJ systems with $m=5$, and (3) mixtures of LJ systems with $m=12$ ( $A$ species $)$ and $m=5$ ( $B$ species $)$. While FCC is the crystalline structure that competes with glass formation for binary LJ systems with $m=12$, BCC is the competing crystalline structure for binary mixtures with $m=5$ for all number fractions $x_{B}$ as shown in Fig. 5. For both $m=12$ and $m=5$ systems, the variation in $R_{c}\left(x_{B}\right)$, which is less than an order of magnitude, is controlled by the diameter ratio $\alpha=0.95$. In binary mixtures of $\mathrm{LJ}$ systems with $m=12$ and $m=5$ interactions, FCC remains the crystalline structure that competes with glass formation, except when $x_{B} \approx 1$. However, because of the incompatibility between FCC and BCC crystalline structures, the GFA for the $m=12$ and $m=5$ LJ mixtures is significantly enhanced compared to glasses with $m=12$ or $m=5$ interactions alone. For example, Ni-Ta is a good glass former despite the fact that it possesses a diameter ratio near unity $(\alpha \approx 0.9)[65]$. Incompatibility between competing BCC and FCC crystal structures is a possible cause of the enhanced GFA. As shown in Table I. Ni has a relatively large pairwise repulsive exponent $(6<m<10)$ with equilibrium FCC structure, while Ta has a relatively small exponent $(3<m<5)$ with equilibrium BCC structure [44]. Since the softness exponents of the pairwise interactions vary significantly from one element to another (Table I), softness-induced competing crystal incompatibility can enhance the GFA of binary and multi-component BMG-forming alloys.

\subsection{Non-additivity}

We performed event-driven molecular dynamics simulations of binary non-additive hard spheres (Sec. 22.2) to investigate the effects of non-additivity of the pairwise repulsive interactions on the GFA of alloys. We measured the critical cooling rate $R_{c}$ of non-additive binary hard spheres with diameter ratios $\alpha=\sigma_{B} / \sigma_{A}=1.0,0.97$, $0.95,0.93,0.9$, and 0.5 and number fractions of the small spheres $x_{B}=0.5$ and $2 / 3$ over a range of non-additivity parameters $\Sigma$. Since $\Sigma>0$ is rare among binary alloys (Table II), we expect that hard-sphere systems with positive non-additivity are poor glass-formers. For example, we find that systems with $\alpha=1$ and $\Sigma=0.05$ display strong demixing between $A$ and $B$ particles and are not good glass formers.

Our previous studies of additive binary hard spheres $(\Sigma=0)$ have shown that well-mixed FCC solid solutions are the crystal structures that compete with glass formation when $\alpha \gtrsim 0.8$, while the systems tend to demix when $\alpha \lesssim 0.8$ [15]. For $\Sigma<0$ and $\alpha=1.0,0.97,0.95,0.93$, and 0.9 , the GFA improves as $\Sigma$ becomes more negative, and the competing crystal structure remains the FCC solid solution (Fig. 6). The change in $R_{c}$ with decreasing $\Sigma$ also increases as $\alpha$ decreases with roughly an order of

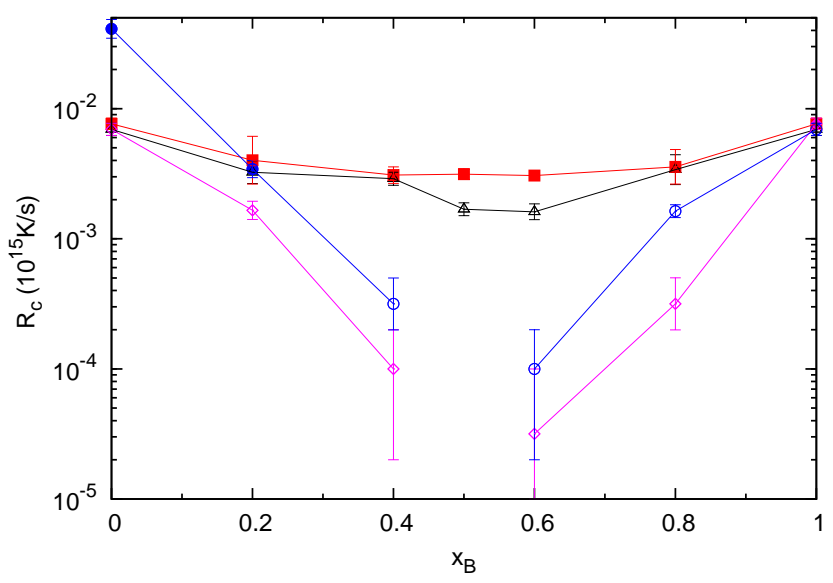

FIG. 5: The critical cooling rate $R_{c}$ for binary mixtures of spherical atoms that interact via the generalized $m-6$ LJ potential (Eq. 1) at diameter ratio $\sigma_{B} / \sigma_{A}=0.95$ is plotted as a function of the number fraction of small atoms $x_{B}$. We show $m=5$ (squares) and 12 (triangles) LJ systems, mixtures (diamonds) of $m=12$ (species $A$ ) and 5 (species $B$ ), as well as mixtures (circles) of spheres with DZ ( $A$ species) and $m=12$ LJ ( $B$ species) interactions. Open and filled symbols indicate that the crystalline structure that competes with glass formation is FCC and BCC, respectively.

magnitude difference in $R_{c}$ between systems with $\Sigma=0$ and $\Sigma=-0.05$ at $\alpha=0.9$. Enhancement of the GFA arising from non-additivity of the repulsive cores $(\Sigma<0)$ has also been observed in LJ systems [66].

For binary systems with large atomic size differences (i.e. $\alpha \ll 0.8$ ), the variation of $R_{c}$ with $\Sigma$ is opposite to that obtained for binary systems with small atomic size differences. As shown in Fig. 6] we find that $R_{c}$ grows with increasing $\Sigma$ at $\alpha=0.5$. For $\alpha=0.5$ and $\Sigma<0$, compound crystals are the ordered structures that compete with glass formation since negative nonadditivity promotes mixing. As an example, although the $A B_{2}$ compound is the densest crystal for binary hard spheres with $\alpha=0.5$ and $\Sigma=0$, it is not kinetically accessible during compression due to the strong drive for demixing [15, 67, 68]. However, when $\Sigma$ becomes negative (e.g. $\Sigma=-0.05$ ), we find that the $A B_{2}$ compound forms easily for the compression rates that we studied, as shown in the inset to Fig. 6. Thus, the formation of intermetallic compounds in alloys can be enhanced by pairwise negative non-additivity among different atomic species.

\subsection{Intermediate-range Repulsive Interactions}

We also investigated crystallization and glass formation as a function of the form of intermediate-range repulsive pairwise interactions (Sec. 22.3). We first performed molecular dynamics simulations of monodisperse spheres interacting via the DZ potential (Eq. 6) at fixed strength 


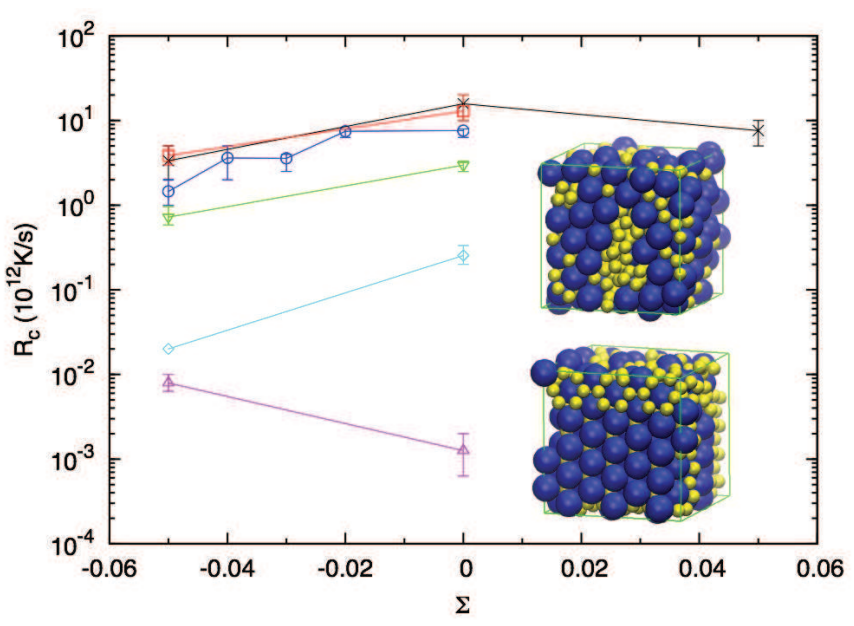

FIG. 6: Critical cooling rate $R_{c}$ (in units of $10^{12} \mathrm{~K} / \mathrm{s}$ ) plotted as a function of the nonadditivity parameter $\Sigma$ for binary hard-sphere systems for several diameter ratio and smallsphere number fraction combinations: $\alpha=1.0$ and $x_{B}=0.5$ (crosses), 0.97 and 0.5 (squares), 0.95 and 0.5 (pentagons), 0.93 and 0.5 (downward triangles), 0.90 and 0.5 (diamonds), and 0.5 and $2 / 3$ (upward triangles). The inset shows snapshots of (top) demixed and (b) compound crystals that form for $R<R_{c}$ at $\Sigma=0.0$ and -0.05 , respectively, with diameter ratio $\alpha=0.5$.

$\xi=0.35 \epsilon$ and varying peak location $(\lambda+\delta) / 2$ and width $\delta-\lambda$. In Fig. 7, we plot the critical cooling rate $R_{c}$ as a contour plot versus $(\lambda+\delta) / 2$ and $\delta-\lambda$ over ranges that are relevant to BMGs (Table III). We find several regions of good glass-forming ability (small $R_{c}$ ) and different crystal structures that compete with glass formation. For a large region of parameter space, FCC is the competing crystal structure. BCC is the competing crystal structure when the location of the peak in $u_{\text {bump }}$ approaches thirdneighbor separations at $r_{i j} \approx \sqrt{3} r_{m}$. We also find an "84" crystal structure that competes with glass formation, with atom positions located on embedded octagons and squares when they are projected into two dimensions. (See the inset of Fig. 7). In three dimensions, one can see that the atoms forming the octagons and squares are located in alternating stacked layers. (See Fig. 8 for a comparison of the radial distribution functions for FCC, BCC, and 8-4 crystals.) When the intermediate-range repulsion becomes too strong (i.e. large $\delta$ ), microphase separation becomes energetically favorable compared to macroscale phase separation [69, 70].

We also studied the critical cooling rate $R_{c}$ for binary mixtures (e.g. Zr-Cu alloys), in which one component possesses intermediate-range repulsive interactions and the other component does not. We focused on binary systems with atoms that interact via the DZ $(A$ species) and LJ potential ( $B$ species) with diameter ratio $\sigma_{B} / \sigma_{A}=0.95$. For the $\mathrm{DZ}$ potential, we set the parameters $\xi / \epsilon \approx 0.4, \lambda \approx 1.2$, and $\delta \approx 2.2$ to mimic those of $\mathrm{Zr}$ atoms (Table III). As shown in Fig. 5. $R_{c}$ for this binary mixture is suppressed by more than two orders

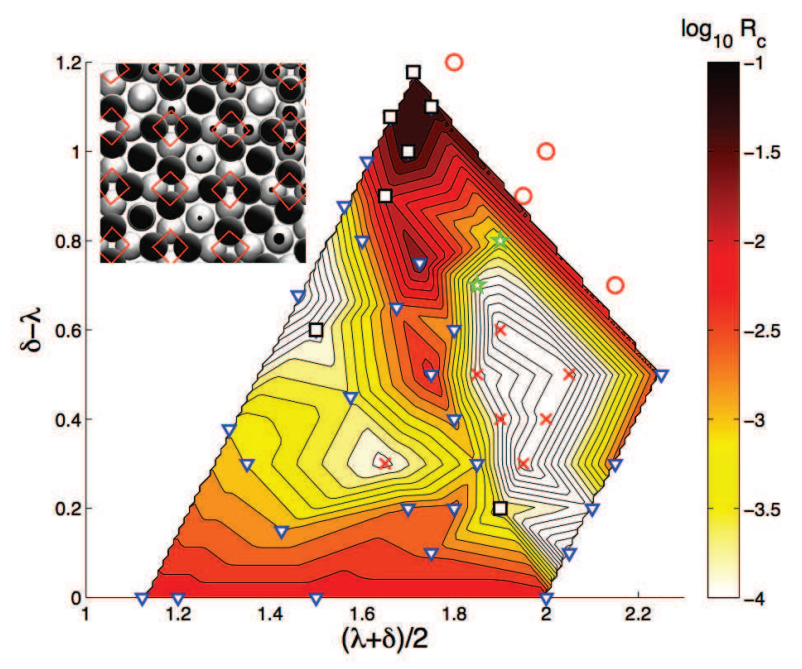

FIG. 7: Contour plot of the critical cooling rate $R_{c}$ (in reduced unit) for monodisperse spheres that interact via the DZ potential (Eq. 6) as a function of the location of the peak $(\lambda+\delta) / 2$ and width $\delta-\lambda$ of $u_{\text {bump. }}$. The bounds for the parameters are determined by $\lambda>r_{m} / \sigma_{A}=1.12$ and $\delta<r_{c} / \sigma_{A}=2.5$. $R_{c}$ contours are interpolated from simulation data points. The symbols indicate where FCC (triangles), BCC (squares), 8-4 (stars) crystalline structures, and microphase separation (circles) is observed. Crosses indicate systems for which the competing crystal structure is unknown and $R_{c}$ is estimated from the slowest cooling rate employed. The inset shows a snapshot of a 8-4 crystal that includes top (dark) and bottom (light) layers of atoms with square symmetry (red squares).

of magnitude compared to the pure system with LJ or DZ interactions alone because the two species possess incompatible equilibrium crystal structures (i.e. FCC and BCC). This mechanism of incompatible equilibrium crystal structures may explain the exceptionally good glassforming ability of the $\mathrm{Zr}-\mathrm{Cu}$ system, even though it is a binary, rather than, multi-component alloy.

\subsection{LJ EAM for Monoatomic Systems}

To determine the relative contributions of the pairwise and many-body interactions to the GFA of alloys, we performed molecular dynamics simulations of the LJ-EAM potential (Sec. 22.4) as a function of the many-body interaction strength $A$ and electron density inverse decay length $\beta$ for monoatomic systems. In Fig. 9, we show the critical cooling rate $R_{c}$ for monodisperse LJ-EAM systems as a function of $A$ for $\beta=2,4$, and $6 \AA^{-1}$. We find that $R_{c} \approx 10^{13} \mathrm{~K} / \mathrm{s} . R_{c}$ changes by less than one order of magnitude as $A$ and $\beta$ are varied over the range that is relevant for elements found in BMGs even though the total potential energy per atom $U / N$ varies linearly with $A$. We also find that FCC crystals are the ordered structures that compete with glass formation in monoatomic LJ-EAM systems over the full parameter range for $A$ and 


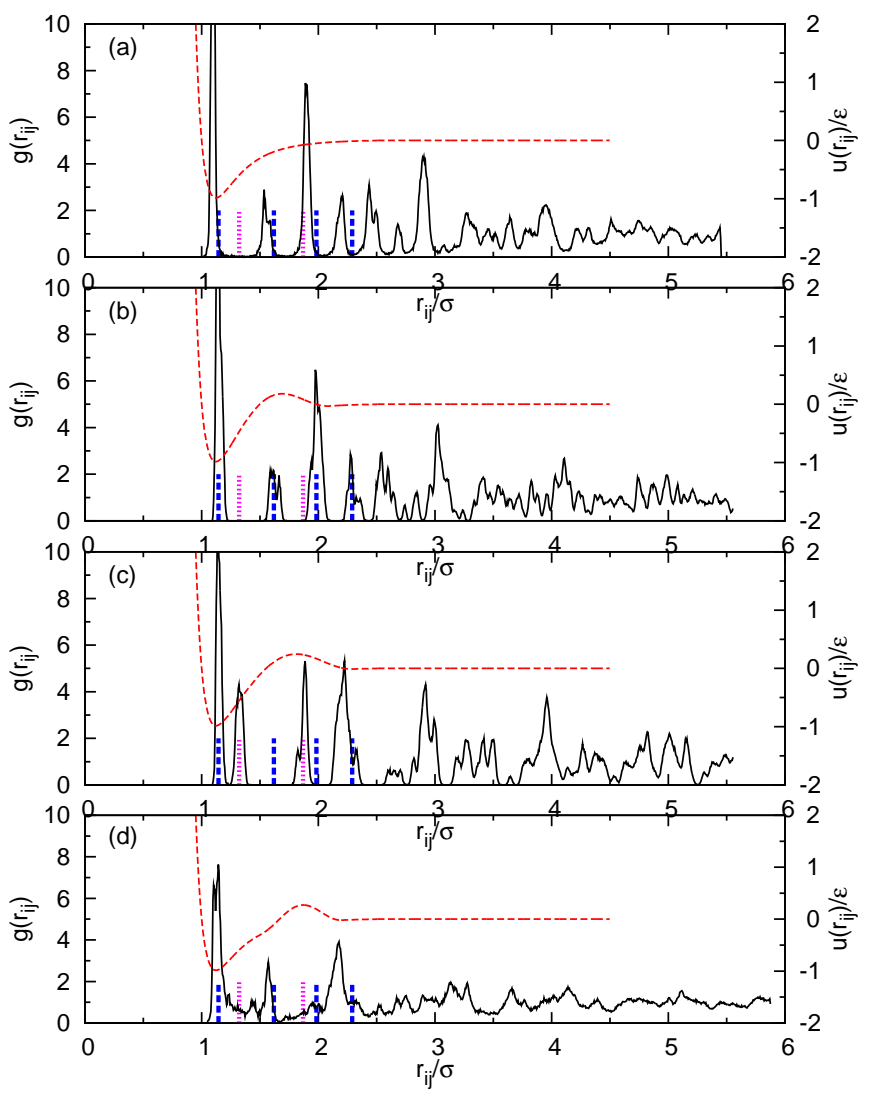

FIG. 8: Radial distribution function $g\left(r_{i j}\right)$ (solid lines, left axis) and pair potential $u\left(r_{i j}\right)$ (dashed lines, right axis) for monodisperse spheres that interact via (a) the Lennard-Jones potential in a FCC crystal structure and via the DZ potential with (b) $\lambda=\sqrt[6]{2}$ and $\delta=2.1$ in a FCC crystal structure, (c) $\lambda=1.2$ and $\delta=2.3$ in a BCC crystal structure, and (d) $\lambda=$ 1.5 and $\delta=2.2$ in a $8-4$ crystal structure. The vertical dotted and dashed lines indicate the BCC lattice spacings (relative to $\left.r_{m}\right) 1: 2 / \sqrt{3}: 2$ and the FCC lattice spacings $1: \sqrt{2}: \sqrt{3}: 2$ up to third and fourth nearest neighbors, respectively.

$\beta$. Thus, we argue that many-body interactions have a weak influence on the GFA compared to the pairwise interactions for monoatomic systems.

\subsection{Full EAM for Binary Alloys}

We also measured the critical cooling rate $R_{c}$ for several binary alloys as a function of the number fraction $x_{B}$ of the small atomic species using the full EAM potential. We focused on $\mathrm{Zr}-\mathrm{Cu}, \mathrm{Mg}-\mathrm{Al}$, and $\mathrm{Cu}-\mathrm{Ni}$ alloys with atomic diameter ratios that range from $\alpha=0.79$ to 0.98. In Fig. 10, we compare $R_{c}$ versus $x_{B}$ from simulations of the full EAM potential for these alloys to $R_{c}$ obtained from simulations of additive hard spheres with comparable values of $\alpha$ [15].

As expected, $R_{c}$ for binary alloys with $\alpha \sim 1$ (i.e. $\mathrm{Cu}-\mathrm{Ni}$ ) is nearly independent of $x_{B}$. In addition, when

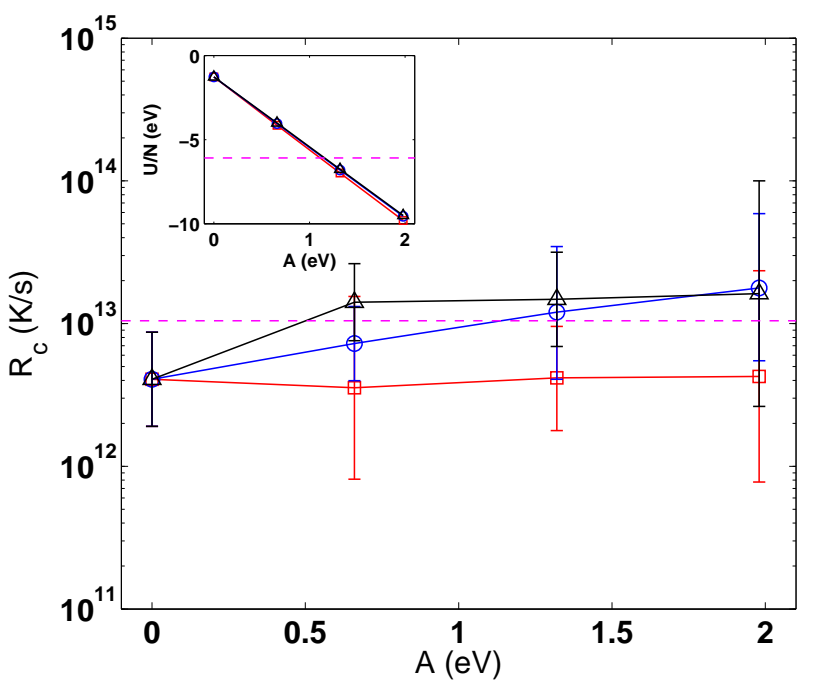

FIG. 9: The critical cooling rate $R_{c}$ from simulations of the LJ-EAM plotted as a function of the many-body interaction strength $A$ (in $\mathrm{eV}$ ) for several values of the electron density inverse decay length $\beta=2$ (squares), 4 (circles), and $6 \AA^{-1}$ (triangles). $R_{c}$ from simulations of the full EAM for $\mathrm{Zr}$ (i.e. $A \approx 1.32 \mathrm{eV}$ and $\beta \approx 4 \AA^{-1}$ ) is indicated by the horizontal dashed line. Error bars give the standard deviation from 10 independent simulations with random initial conditions. The inset shows the total potential energy $U / N$ per atom (for cooling rates $R>R_{c}$ ) versus $A$ for the same values of $\beta$ in the main panel. $U / N$ for the full EAM of $\mathrm{Zr}$ is given by the horizontal dashed line.

the hard-sphere simulations with $\alpha=1$ are calibrated to $\mathrm{Ni}, R_{c}$ from simulations of the hard-sphere and EAM potentials agree semi-quantitatively. From our previous simulations of hard spheres [15], we know that $R_{c}\left(x_{B}\right)$ develops a deep minimum that shifts to larger $x_{B}$ as $\alpha$ decreases from unity. For example, when $\alpha=0.9, R_{c}$ for hard-sphere systems at $x_{B} \approx 0.6$ is two orders of magnitude less than the value when $\alpha=1$. Although we are not able to simulate sufficiently slow rates, it appears that $R_{c}$ at the minimum in $x_{B}$ for $\mathrm{Mg}$ - $\mathrm{Al}$ with $\alpha=0.94$ will decrease by at least two orders of magnitude and the minimum in $R_{c}\left(x_{B}\right)$ will occur at $x_{B}>0.5$. We also find similar results for $R_{c}$ for hard spheres with $\alpha=0.79$ and for $\mathrm{EAM}$ of $\mathrm{Zr}-\mathrm{Cu}$ with a deep minimum in the range $0.2<x_{B}<0.8$.

We also determined the crystal structures that compete with glass formation in the full EAM simulations of binary alloys. We find that FCC (or HCP) is most often the competing crystal structure, as in simulations of additive binary hard spheres, but we also find exceptions. In particular, we show that on the Zr-rich side of $\mathrm{Zr}-\mathrm{Cu}, \mathrm{BCC}$ crystal structures compete with glass formation. The BCC equilibrium structure for the $\mathrm{Zr}-\mathrm{Cu}$ alloys can likely be attributed to the pairwise part of the EAM potential. For example, the pair potential for $\mathrm{Zr}$ possesses intermediate-range repulsive interactions with the 


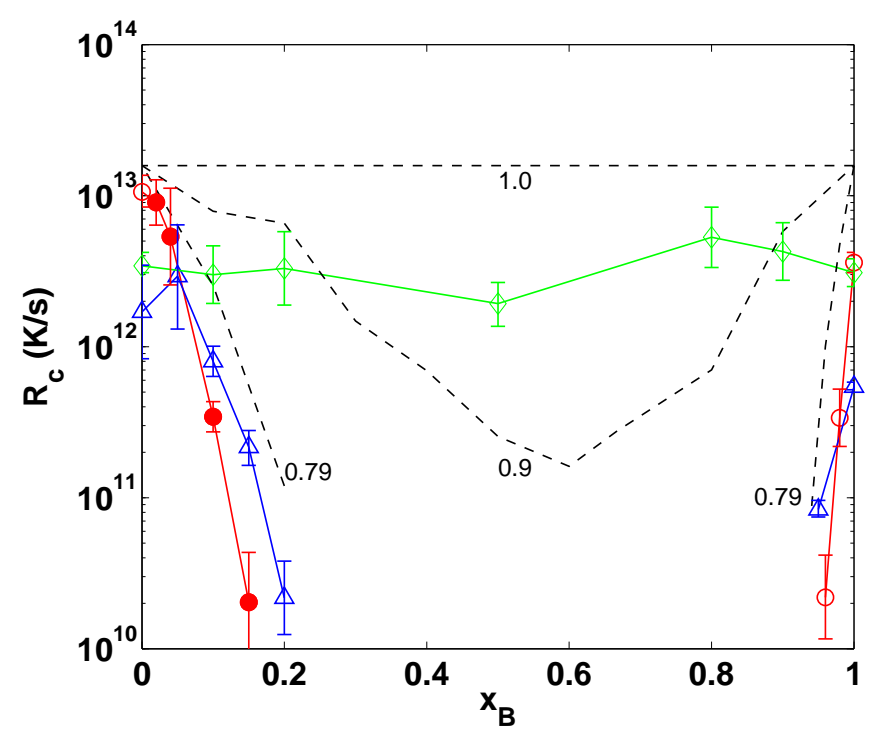

FIG. 10: The critical cooling rate $R_{c}$ (in $\mathrm{K} / \mathrm{s}$ ) for several binary alloys, including $\mathrm{Zr}-\mathrm{Cu}$ with atomic diameter ratio $\alpha=0.79$ (circles), $\mathrm{Mg}$-Al with $\alpha=0.94$ (triangles), and $\mathrm{Cu}-\mathrm{Ni}$ with $\alpha=0.98$ (diamonds), is plotted as a function of number fraction of small atoms $x_{B}$ using molecular dynamics simulations of the full EAM. Error bars on $R_{c}$ are obtained from the standard deviation from 5 independent simulations. The EAM source files are given in Refs. 29, 34, 71 73. As a comparison, $R_{c}$ for additive binary hard spheres with $\alpha=1.0$, 0.9 , and 0.79 are shown as dashed lines. We also indicate when FCC or HCP (open symbols) and BCC (filled symbols) crystal structures compete with glass formation.

location of peak $(\lambda+\delta) / 2=1.70$ and width $\delta-\lambda=1.08$ (Table III) in a region of parameter space that has been shown to display BCC crystal structure (Fig. 17).

\section{CONCLUSION}

The hard-sphere model has provided a predictive description of crystallization and glass formation in simple liquids [33]. In addition, we have shown in recent studies that the additive hard-sphere model can explain more than 13 orders of magnitude variation in the critical cooling rate $R_{c}$, which nearly spans the full range of GFA from that for pure metals to that for the best BMGs [15]. We also showed that the best binary and ternary BMGs occur in the region of parameter space (i.e. diameter ratio and number fraction) with the smallest values of $R_{c}$ for hard spheres.

However, in metallic systems, there are a number of additional features of the interatomic potential beyond hard-core repulsions, including softness, non-additivity, and range of the pairwise interactions. For example, metallic atoms typically appear softer (with smaller values of the exponent of the repulsive core) than the commonly used LJ pair potential and possess several per cent negative non-additivity due to shortening of metallic bonds 47. In addition, Friedel oscillations in metals give rise to intermediate-range repulsion at separations beyond the short-range attractive well [52]. The interatomic potential for metals also includes many-body interactions from the electronic degrees of freedom. In this manuscript, we investigated how these additional features affect the GFA of pure and binary metallic systems.

We performed molecular dynamics simulations of several model systems to study the effects on the GFA for each of the key features of the interatomic potential separately. For example, we performed simulations of monodisperse and binary spheres that interact via the generalized LJ and DZ pair potentials to quantify the effect of the softness of the repulsive core and form of the intermediate-range repulsive interactions on the GFA. We also performed MD simulations of non-additive binary hard spheres to quantify the effects of non-additivity on the GFA. We found that softness, non-additivity, and form of the intermediate-range repulsions cause deviations in $R_{c}$ that are only $1 \sim 2$ orders of magnitude from the additive hard-sphere predictions.

While FCC is the most stable crystal structure for LJ and hard-sphere systems, softening of the repulsive core gives rise to novel contracted disordered structures, as well as the formation of BCC crystals. We also showed that negative non-additivity of the repulsive core in binary alloys improves the GFA when the competing crystal structures are solid solutions. However, when the atomic size ratio is in the demixing regime $(\alpha<0.8)$, negative non-additivity can favor the formation of compound crystals and decrease the GFA. The crystal structure that competes with glass formation, and thus the GFA, also depends sensitively on the form of the intermediate-range repulsive interactions. We find that when the competing crystal structures of each component in an alloy are incompatible (e.g. FCC and BCC), the GFA can be enhanced compared to hard-sphere predictions.

We also investigated the relative contributions of the pairwise and many-body interactions to the GFA by performing molecular dynamics simulations of the LJ-EAM potential. We found that including the many-body interactions only changes $R_{c}$ by less than one order of magnitude compared to that when the many-body interactions are not included. We also calculated $R_{c}$ for several binary alloys using the full EAM potential and found qualitatively the same results as for binary hard spheres. Thus, we argue that hard-sphere interactions provide a qualitatively accurate model for predicting the GFA of alloys. Other features of the interatomic potential (beyond additive hard-core repulsion) give rise to only 1-2 orders of magnitude variation of $R_{c}$, which is small compared to the more than 13 orders of magnitude variation predicted by hard-sphere systems. Despite this, including additional features to the interatomic potential beyond hard-sphere interactions is important for the design of new BMGs since precise quantification of the critical casting thickness can determine whether a new BMG is 
commercially viable.

\section{Acknowledgments}

The authors acknowledge primary financial support from the NSF MRSEC DMR-1119826 (KZ) and partial support from NSF grant numbers CMMI-1462439 (CO, MF) and CMMI-1463455 (MS). This work also benefited from the facilities and staff of the Yale University Faculty of Arts and Sciences High Performance Computing Center and the NSF (Grant No. CNS-0821132) that in part funded acquisition of the computational facilities.
[1] M. Telford, Materials Today 7, 36 (2004).

[2] J. Schroers, Physics Today 66, 32 (2013).

[3] L. Zhong, J. Wang, H. Sheng, Z. Zhang, and S. X. Mao, Nature 512, 177 (2014).

[4] A. L. Greer, Science 267, 1947 (1995).

[5] C. Suryanarayana and A. Inoue, Bulk Metallic Glasses (CRC Press, New York, 2011).

[6] P. Tsai and K. M. Flores, Intermetallics 55, 162 (2014).

[7] S. Ding, Y. Liu, Y. Li, Z. Liu, S. Sohn, F. J. Walker, and J. Schroers, Nature Materials 13, 494 (2014).

[8] K. Zhang, B. Dice, Y. Liu, J. Schroers, M. D. Shattuck, and C. S. O'Hern, J. Chem. Phys. 143, 054501 (2015).

[9] P. J. Steinhardt, D. R. Nelson, and M. Ronchetti, Phys. Rev. B 28, 784 (1983).

[10] W. Klement, R. H. Willens, and P. Duwez, Nature 187, 869 (1960).

[11] P. G. Debenedetti, J. Phys.: Condens Matter 15, R1669 (2003).

[12] J. Schroers, Nature 512, 142 (2014).

[13] M. C. Weinberg, D. R. Uhlmann, and E. D. Zanotto, J. Am. Ceram. Soc. 72, 2054 (1989).

[14] Y. Yokoyama, N. Nishiyama, K. Fukaura, H. Sunada, and A. Inoue, Mater. Trans., JIM 40, 696 (1999).

[15] K. Zhang, W. W. Smith, M. Wang, Y. Liu, J. Schroers, M. D. Shattuck, and C. S. O'Hern, Phys. Rev. E 90, 032311 (2014).

[16] W. H. Zachariasen, J. Am. Chem. Soc. 54, 3841 (1932).

[17] W. L. Johnson, MRS bulletin 24, 42 (1999).

[18] R. S. Hoy and C. S. O'Hern, Soft Matter 8, 1215 (2012).

[19] T. Egami and Y. Waseda, J. Non-Cryst. Solids 64, 113 (1984).

[20] D. B. Miracle, W. S. Sanders, and O. N. Senkov, Philos. Mag. 83, 2409 (2003).

[21] D. B. Miracle, Nature Materials 3, 697 (2004).

[22] D. B. Miracle, Acta Mater. 61, 3157 (2013).

[23] P. Jalali and M. Li, Intermetallics 12, 1167 (2004).

[24] P. Jalali and M. Li, Phys. Rev. B 71, 014206 (2005).

[25] K. Zhang, Y. Liu, J. Schroers, M. D. Shattuck, and C. S. O'Hern, J. Chem. Phys. 142, 104504 (2015).

[26] M. S. Daw and M. I. Baskes, Phys. Rev. Lett. 50, 1285 (1983).

[27] A. Inoue, Acta Mater. 48, 279 (2000).

[28] M. I. Mendelev and G. J. Ackland, Philos. Mag. Lett. 87, 349 (2007).

[29] H. W. Sheng, M. J. Kramer, A. Cadien, T. Fujita, and M. W. Chen, Phys. Rev. B 83, 134118 (2011).

[30] M. Dzugutov, Phys. Rev. A 46, R2984 (1992).

[31] Y. Shi, J. Luo, F. Yuan, and L. Huang, J. Appl. Phys. 115, 043528 (2014).

[32] S. Zhen and G. Davies, Phys. Stat. Sol. (a) 78, 595 (1983).

[33] J. D. Weeks, D. Chandler, and H. C. Andersen, J. Chem.
Phys. 54, 5237 (1971).

[34] X. W. Zhou, R. A. Johnson, and H. N. G. Wadley, Phys. Rev. B 69, 144113 (2004).

[35] G. P. P. Pun and Y. Mishin, Phys. Rev. B 86, 134116 (2012).

[36] P. L. Williams, Y. Mishin, and J. C. Hamilton, Modelling Simul. Mater. Sci. Eng. 14 (2006).

[37] R. Ravelo, T. C. Germann, O. Guerrero, Q. An, and B. L. Holian, Phys. Rev. B 88, 134101 (2013).

[38] Y. Li, D. J. Siegel, J. B. Adams, and X.-Y. Liu, Phys. Rev. B 67, 125101 (2003).

[39] J. M. Winey, A. Kubota, and Y. M. Gupta, Modelling Simul. Mater. Sci. Eng. 17 (2009).

[40] G. Grochola, S. P. Russo, and I. K. Snook, J. Chem. Phys. 123, 204719 (2005).

[41] Y. Mishin, D. Farkas, M. J. Mehl, and D. A. Papaconstantopoulos, Phys. Rev. B 59, 3393 (1999).

[42] M. R. Fellinger, H. Park, and J. W. Wilkins, Phys. Rev. B 81, 144119 (2010).

[43] M. P. Allen and D. J. Tildesley, Computer Simulation of Liquids (Oxford University Press, New York, 1987).

[44] W. Hume-Rothery, The Structure of Metals and Alloys (The Institute of Metals, London, 1950).

[45] S. Punnathanam and P. Monson, J. Chem. Phys. 125, 024508 (2006).

[46] L. V. Woodcock, Ind. Eng. Chem. Res. 50, 227 (2011).

[47] Y. Q. Cheng, E. Ma, and H. W. Sheng, Phys. Rev. Lett. 102, 245501 (2009).

[48] O. Senkov, Y. Cheng, D. Miracle, E. Barney, A. Hannon, and C. Woodward, J. Appl. Phys. 111, 123515 (2012).

[49] H. W. Sheng, W. K. Luo, F. M. Alamgier, J. M. Bai, and E. Ma, Nature 439, 419 (2006).

[50] W. Kob and H. C. Andersen, Phys. Rev. E 51, 4626 (1995).

[51] T. M. Truskett, S. Torquato, and P. G. Debenedetti, Phys. Rev. E 62, 993 (2000).

[52] J. Friedel, Il Nuovo Cimento 7, 287 (1958).

[53] N. Fujima, M. Asato, R. Tamura, and T. Hoshino, Materials Transactions 48, 1734 (2007).

[54] N. Wu, D. Kan, L. Zuo, and J. Wang, Intermetallics 39, 1 (2013).

[55] M. Dzugutov, Phys. Rev. Lett. 70, 2924 (1993).

[56] J. Roth and A. R. Denton, Phys. Rev. E 61, 6845 (2000).

[57] J. P. K. Doye and D. J. Wales, Phys. Rev. Lett. 86, 5719 (2001).

[58] M. Baskes, Phys. Rev. Lett. 83, 2592 (1999).

[59] H.-S. Nam, M. Mendelev, and D. Srolovitz, Phys. Rev. B 75, 014204 (2007).

[60] S. Plimpton, J. Comput. Phys. 117, 1 (1995).

[61] G. Parisi and F. Zamponi, Rev. Mod. Phys. 82, 789 (2010).

[62] W. G. Hoover, D. A. Young, and R. Grover, J. Chem. 
Phys. 56, 2207 (1972)

[63] D. Frenkel, Science 314, 768 (2006).

[64] H. W. Sheng, H. Z. Liu, Y. Q. Cheng, J. Wen, P. L. Lee, W. K. Luo, S. D. Shastri, and E. Ma, Nature 6, 192 (2007).

[65] Y. Wang, Q. Wang, J. Zhao, and C. Dong, Scripta Materialia 63, 178 (2010).

[66] K. Zhang, M. Wang, S. Papanikolaou, Y. Liu, J. Schroers, M. D. Shattuck, and C. S. O'Hern, J. Chem. Phys. 139, 124503 (2013).

[67] L. Filion and M. Dijkstra, Phy. Rev. E 79, 046714 (2009).
[68] A. B. Hopkins, Y. Jiao, F. H. Stillinger, and S. Torquato, Phy. Rev. Lett. 107, 125501 (2011).

[69] S. Brazovskii, Zh. Eksp. Teor. Fiz 68, 175 (1975).

[70] M. Seul and D. Andelman, Science 267, 476 (1995).

[71] S. M. Foiles, Phys. Rev. B 32, 7685 (1985).

[72] X.-Y. Liu and J. B. Adams, Acta Materialia 46, 3467 (1998)

[73] M. I. Mendelev, M. Asta, M. Rahman, and J. Hoyt, Philosophical Magazine 89, 3269 (2009). 